\title{
Interference relationships in grasshopper reciprocal translocation heterozygotes
}

\author{
P. Arana, \\ J. L. Santos and \\ N. Henriques-Gil*
}

\author{
Departamento de Genética, Facultad de Biología, \\ Universidad Complutense, 28040 Madrid, Spain.
}

Chiasma formation in four different reciprocal translocations in the grasshopper Eyprepocnemis plorans $(2 n \delta=22+$ $\boldsymbol{X}$ ) is analysed using $\mathbf{C}$-banding. A given chromosome involved in an interchange is sometimes identifiable in standard homozygotes so that changes in its formation of chiasmata may be estimated. The involvement of M9 chromosome in an interchange slightly reduces its mean chiasma frequency but interference is significatively reduced too. When interference relationships between arms are analysed in the interchanges, independence as well as interference may occur, the interference being positive between adjacent arms and negative between opposite arms. $\mathbf{A}$ parallel analysis at pachytene reveals synaptic failure affecting mainly small regions. These results are discussed under Sybenga's (1970) model for interference relationships within quadrivalents.

\section{INTRODUCTION}

The effect of reciprocal translocation heterozygosity on chiasma formation in rearranged chromosomes has been analysed in a number of cases both in plants and animals, but the great variety of results obtained has led to no clear idea about which are the meiotic processes affected by the rearrangement. Three aspects of chiasma formation have mainly received attention: the total frequency of chiasmata within the interchange complex, the position of these chiasmata, and the interference relationships that may be established between different regions of the quadrivalent. Considering the interchange as a whole, increases in chiasma frequency (Hewitt, 1967; Arana et al., 1980; Parker et al., 1982), decreases (Rickards, 1964; Southern, 1967) and lack of effect (Bloom, 1974; Gosálvez et al., 1982; Díez and Puertas, 1984) have been reported when the translocation mean chiasma frequency was compared to that of the corresponding two pairs of non-homologous chromosomes in standard homozygotes. On the other hand, when comparisons were carried out between specific segments, the results showed that the response of the different regions may not be

\footnotetext{
* Laboratorio de Genética, Colegio Universitario San Pablo C.E.U. Montepríncipe, Boadilla del Monte, Madrid, Spain.
}

uniform (man: Holm and Rasmussen, 1978; mouse: de Boer and van Beek, 1982) as in the grasshopper interchange of Euchorthippus involving the L3 and M6 chromosomes described by Arana et al. (1980). In this interchange the L3 chromosome did not show any difference in chiasma frequency compared with standard homozygotes, whereas the M6 chromosome showed a significant increase, accompanied by reduction in interference between regions separated by the breakpoint, and an increase in the localisation of chiasmata in its most distal segment.

On the other hand, using genetical methods, a typical reduction in recombination frequency around the breakpoint has been demonstrated (Eicher and Green, 1972; Hawley, 1980; Burnham, 1981), that may be accompanied by an increase somewhere else within the interchange.

With respect to the interference relationships established between the different quadrivalent arms, in some cases a total independence has been found (Sybenga, 1975; Arana et al., 1980); the breakpoint seems to act as a barrier to interference. However, John and Hewitt (1963) claimed the existence of positive interference between the interstitial and translocated segments of one of the chromosomes involved in a Chorthippus interchange and Jost and Laven (1971) explained the configurations obtained in a Culex translocation 
by postulating that all arms of this interchange showed positive interference.

Negative interference between specific regions of interchanges has been also reported (Sybenga, 1975; de Boer and van der Hoeven, 1977) and this has been subsequently tested in a number of different translocations in rye (Sybenga and Mastenbroek, 1980) and wheat (Fominaya and Jouve, 1986). According to Sybenga's model (Sybenga, 1970; 1975) there should be negative interference between segments located in opposite arms of the pachytene cross and positive interference between adjacent ones. This pattern of interference is explained by anomalous pairing in the interchange complex.

However, it is important to notice that not all metaphase I meiotic configurations analyzed in the studies in cereals could be unambiguously identified, which makes it necessary to estimate interference by indirect methods. It is also possible that in cases where telocentric chormosomes are involved, interference relationships may be established in a different way because there are no non-translocated arms. To date, Sybenga's model has been never tested for telocentric chromosomes.

In the grasshopper interchanges studied here both the segment lengths and the C-banding patterns of chromosomes have allowed reliable identification of the four arms of each pachytene cross and thus accurate estimates of the actual frequency of chiasmata at diplotene in each arm. Thus when a chromosome can be identified in standard homozygotes and translocation heterozygotes it is possible to analyse the effect of the rearrangement on chiasma formation. In addition, the interference relationships can be estimated by applying simple statistical tests to the diplotene observations. Our results are interpreted under Sybenga's model for interference in quadrivalents and some possible causes to account for the variation found are proposed.

\section{MATERIAL AND METHODS}

Four different reciprocal translocations of Eyprepocnemis plorans $(2 n \delta=22+X, 2 n \uparrow=22+$ $X X$ ) have been cytologically analysed by means of C-banding. All the chromosomes in this species are telocentric and show positive C-heterochromatin in the centromeric regions. The features of three of these interchanges (A1, E2411 and E1921) have been described in a previous paper (Arana et al., 1987). Briefly, A1 is a spontaneous translocation involving one of the longest pairs of the complement ( $\mathrm{L}$ group) and the chromosome M9. Two more are X-ray induced: E2411 involves a long (L group) and a medium (M group) chromosomes and in E1921 the longest chromosome (L1) and one medium pair carrying a thin pericentric C-band are involved. Of the latter, in addition to the original mutant, three males of its offspring, heterozygous for the same interchange (U3, U9 and U11) were also analysed.

The fourth interchange E1911 is also X-ray induced. It involves two medium ( $M$ group) chromosomes, one of them bearing a pericentric C-band. Figs. 1(a)-(e) show the pachytene configurations corresponding to the interchanges described above. In all interchanges regions I and III correspond to interstitial segments and II and IV to translocated ones. The C-banding pattern allowed the identification of the four arms of the pachytene crosses in all cases, and consequently the frequency of chiasmata at diplotene in each of the four regions could be scored unequivocally. Fig. 2 shows some of the diplotene configurations found in the interchanges.

\section{RESULTS}

\section{Diplotene}

Interchange $A 1$. The diplotene configurations and the chiasma frequencies in each of the distinguishable regions of this interchange are shown in table 1. Two types of chains can appear, either open in an interstitial region (type II chains) or in a translocated region (type I chains), the former being much the more frequent. The only segment able to form more than one chiasma is the long interstitial region $\mathrm{III}$, the remaining three may be open ( 0 chiasmata) or closed by a single chiasma. A fair agreement between segment length and chiasma frequency is observed. Nevertheless, the normal tendency towards a distal localisation of chiasmata in this species seems to be roughly maintained since region IV (approximately the distal half of chromosome M9-see fig. 1(a) shows a mean chiasma frequency higher than region I (approximately, the proximal half of chromosome M9).

The analysis of interference was carried out directly on the diplotene observations, applying $\chi^{2}$ contingency tests for each pair of distinguishable regions. Translocated segment II could not be included in this analysis because it did not show enough variation to apply the statistical test. The results are shown in table 1 . Only when regions I 


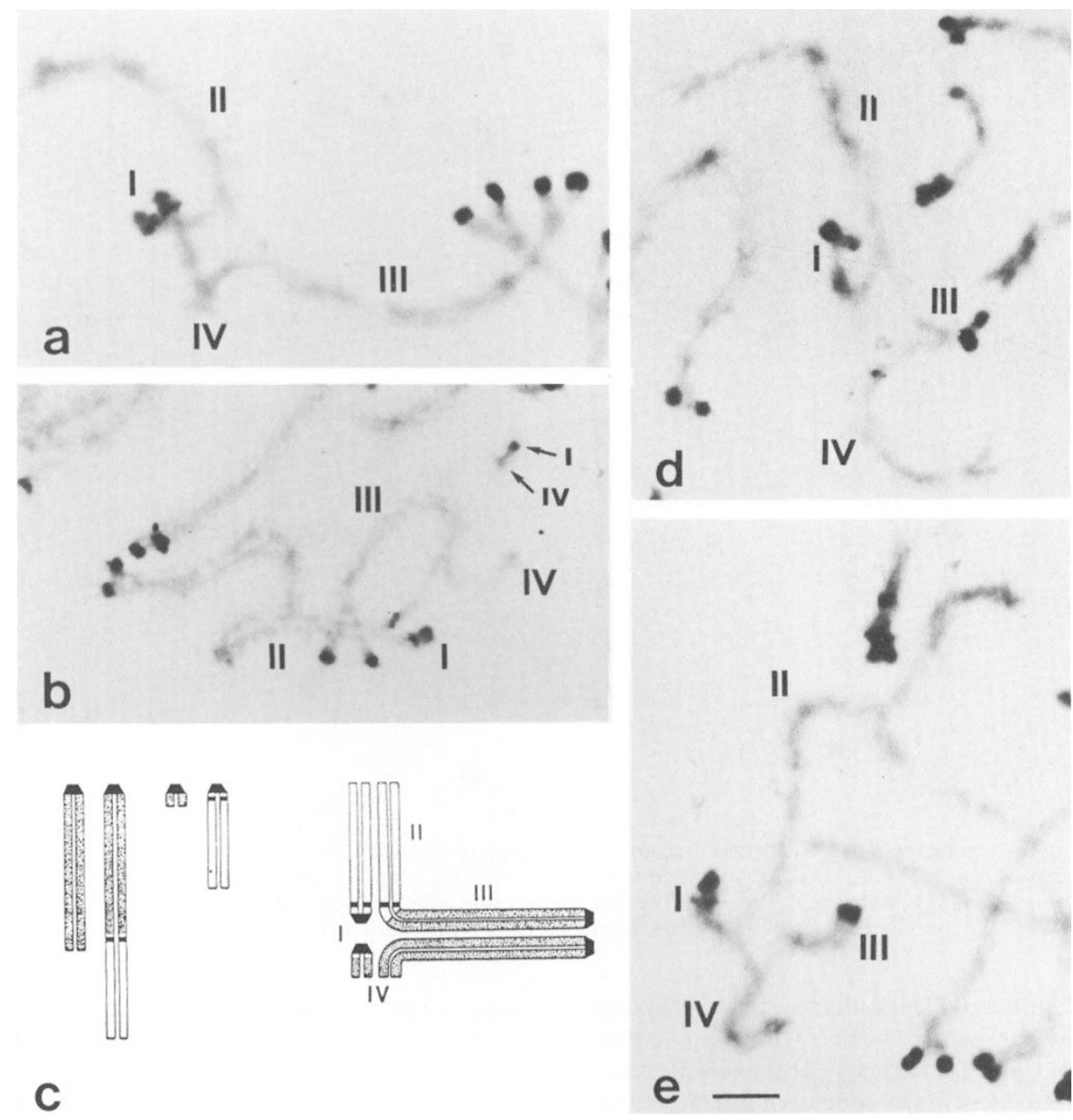

Figure 1 Pachytene configurations of the interchanges and distinguishable regions: 1(a) A1 1(b) E.1911. In this cell there is a failure of pairing in regions I and IV, thus, the small translocated $M$ chromosome appears as an univalent. Arrows point the correspondent pairing regions within this chromosome. 1(c) Scheme of the chromosomes involved in the E.1911 translocation and pachytene pairing cross. 1(d) E.2411. 1(e) E.1921. The paracentric C-band in the M chromosome makes regions I and III distinguishable. In all cases Bar represents $5 \mu \mathrm{m}$.

and IV-interstitial and translocated segments respectively-were tested was a significant $\chi^{2}$ value obtained. This result means that these two regions are not independent of each other with respect to chiasma formation. An excess of the classes in which one region is open and the other closed is observed (positive interference).

Interchange E1911. Table 2 shows the diplotene configurations, chiasma frequencies and $\chi^{2}$ tests for interference in the different regions of this interchange. As region $\mathrm{I}$ is always open, no rings were formed, trivalents and type II chains being the most frequent configurations. Again, a certain correspondence between region length and mean number of chiasmata is found. In this interchange the $\chi^{2}$ contingency test could only be performed between the translocated segments II and IV, giving a non-significant value.

Interchange E2411. In this interchange, regions II and IV always form one chiasma, and so only the short interstitial segments are variable (table 3 ). The $\chi^{2}$ test between these two regions 


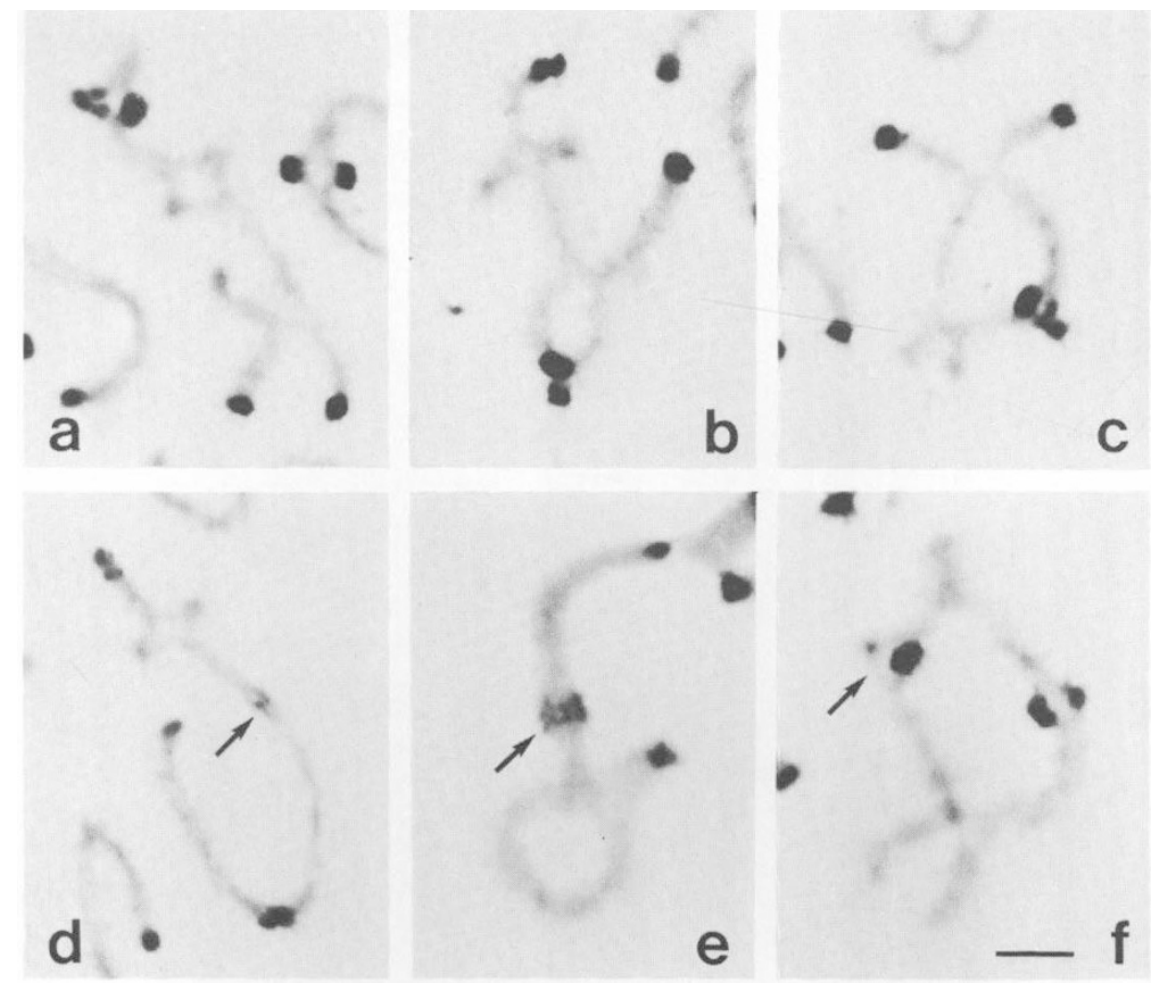

Figure 2 Diplotene configurations: 2(a) A1 type I chain. 2(b) Al type II chain. 2(c) Al ring. 2(d) E.1911 type II chain. Arrow points interstitial position of the originally paracentric C-band. 2(e) E.1921 type II chain open in region III. Arrow points the chiasma in region 1. 2(f) E.2411 ring. Arrow points the chiasma in region I. Note the quantitative difference in C-heterochromatin content between the two centromeres of this region. In all cases Bar represents $5 \mu \mathrm{m}$.

showed a significant deviation towards an excess of the classes in which both regions are either closed or open simultaneously, hence to an excess of rings and bivalent pairs (negative interference).

Interchange E1921. This interchange was analysed in four individuals: the original mutantE1921-and three males of its offspring-U3, U9 and U11. As shown in table 4, the mean chiasma frequency in region IV approaches 1 in all cases so that it was not included in the interference tests. It is noticeable that even though the length of both interstitial regions $\mathrm{I}$ and $\mathrm{III}$ is very similar, the latter consistently shows a higher frequency of chiasmata than the former. The $\chi^{2}$ tests reveal a variety of results: from complete independence between each pair of regions in U9 to the existence of simultaneous positive and negative interference in U3. In two males, E1921 and U11, only negative interference between interstitial regions is detected.

Summarising, when significant deviations from independence between regions in the formation of chiasmata are found, the trend is towards positive interference when the regions tested are adjacent in the pachytene cross whereas negative interference appears between opposite regions. This pattern of interference relationships within interchanges was first described by Sybenga (1970; 1975 ) in rye. The hypothesis proposed by this author is based on the existence of pairing difficulties around the breakpoint in the rearranged chromosomes in such a way that: "... pairing initiated at a number of points along the chromosome proceeds zipper-wise but may not be expected to reach the breakpoint simultaneously from all four directions. When in one segment pairing is complete up to the breakpoint, the adjacent segments may experience considerable torsion which prevents their effective pairing into the direction of the breakpoint. This, then, could be the cause of positive interference between adjacent segments. Since no rigid structure can now be formed around the breakpoint, the remaining segment (opposite that associated first) may have a somewhat better opportunity to associate towards the breakpoint..." (leading to negative interference). 
Table 1 Diplotene configurations, chiasma frequency, and $\chi^{2}$ contingency tests for interference in interchange A1

\begin{tabular}{|c|c|c|c|c|c|c|c|}
\hline \multicolumn{8}{|c|}{ Region } \\
\hline & I & II & III & IV & No. Xta & Configuration & Frequency \\
\hline & - & 1 & 1 & 1 & 3 & chain II & 229 \\
\hline & - & 1 & 2 & 1 & 4 & chain II & 15 \\
\hline & 1 & 1 & 1 & - & 3 & chain I & 39 \\
\hline & 1 & 1 & 2 & - & 4 & chain I & 2 \\
\hline & - & 1 & 1 & - & 2 & III + I & 78 \\
\hline & - & 1 & 2 & - & 3 & III + I & 4 \\
\hline & 1 & 1 & 1 & 1 & 4 & ring & 48 \\
\hline & 1 & 1 & 2 & 1 & 5 & ring & 2 \\
\hline & 1 & - & 1 & 1 & 3 & chain I & 1 \\
\hline & 1 & 1 & - & 1 & 3 & chain I & 1 \\
\hline Mean & $0 \cdot 222$ & 0.998 & 1.053 & 0.706 & 2.979 & & 419 \\
\hline & 0.173 & 0.002 & 0.005 & 0.208 & $0 \cdot 361$ & & \\
\hline S.E. & 0.020 & 0.002 & 0.011 & 0.022 & 0.029 & & \\
\hline
\end{tabular}

Interference Regions

$$
\begin{array}{ll}
\text { I/II } & \chi^{2}=0.125,0.75>p>0.50 \\
\text { I/IV } & \chi^{2}=12.510, p<0.005, \text { Positive interference. } \\
\text { III/IV } & \chi^{2}=0.125,0.75>p>0.50
\end{array}
$$

\section{Pachytene}

In order to test this hypothesis, a qualitative analysis at pachytene was carried out in the interchanges. Lack of pairing around the breakpoint was always observed but only the cells in which any region was totally unpaired were scored. As a result that only the short regions were taken into account since long regions never showed complete unpairing. In E1911 region I was never paired at pachytene but a detailed analysis could not be performed. In the remaining translocations unpaired regions were observed at variable frequencies (table 5(a)-(c)).

It is important to notice that pachytene stage that allows in combination with C-banding a reliable identification of the different chromo- somes may be too late to ascertain whether the lack of pairing is attributable to asynapsis or desynapsis. Nevertheless it should be an intrinsic characteristic of the interchange since the remaining bivalents of the complement appear normally paired (fig. 3a, b).

\section{DISCUSSION}

\section{Changes in chiasma frequency and distribution in the chromosomes involved in the interchanges}

In the Elorans translocations studied here, only the M9 chromosome can be readily identified in

Table 2 Diplotene configurations, chiasma frequency, and $\chi^{2}$ contingency tests for interference in interchange E1911

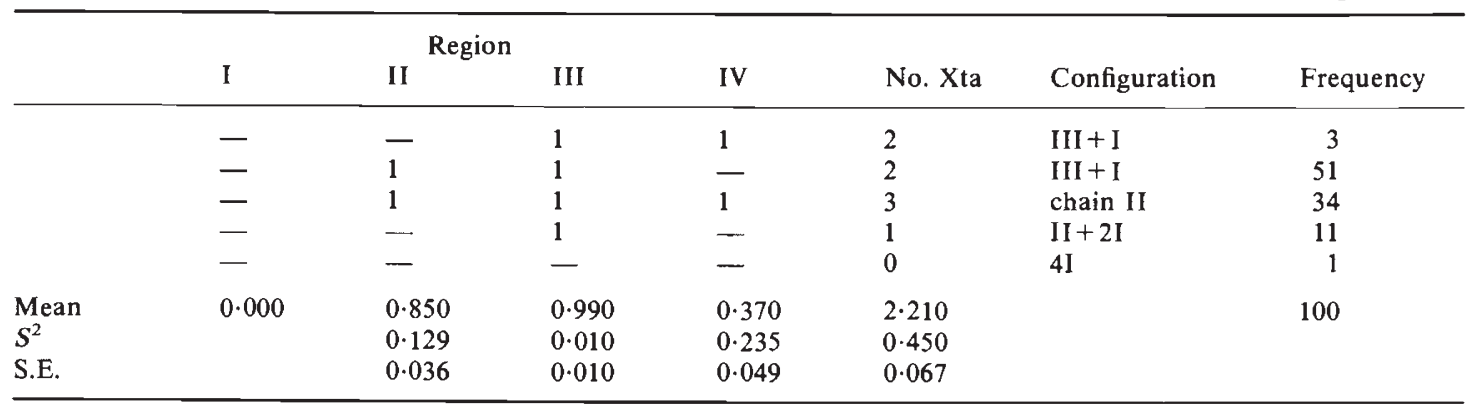

Interference Regions

$$
\text { II } / \text { IV } \quad \chi^{2}=2 \cdot 188,0 \cdot 20>p>0 \cdot 10
$$


Table 3 Diplotene configurations, chiasma frequency and $\chi^{2}$ contingency tests for interference in interchange E2411

\begin{tabular}{|c|c|c|c|c|c|c|c|}
\hline \multicolumn{8}{|c|}{ Region } \\
\hline & 1 & II & III & IV & No. Xta & Configuration & Frequency \\
\hline & - & 1 & - & 1 & 2 & 211 & 90 \\
\hline & - & 1 & 1 & 1 & 3 & chain I & 20 \\
\hline & 1 & 1 & - & 1 & 3 & chain II & 11 \\
\hline & 1 & 1 & 1 & 1 & 4 & ring & 13 \\
\hline Mean & $0 \cdot 179$ & 1.000 & $0 \cdot 246$ & 1.000 & $2 \cdot 425$ & & 134 \\
\hline & $0 \cdot 148$ & & $0 \cdot 187$ & & 0.442 & & \\
\hline S.E. & 0.033 & & 0.037 & & 0.057 & & \\
\hline
\end{tabular}

Interference Regions

I/III $\chi^{2}=13 \cdot 75, p<0.005$ Negative interference.

Table 4 Chiasma frequency and interference relationships between interchange arms in four heterozygotes carrying the 1921 translocation

\begin{tabular}{|c|c|c|c|c|c|c|c|c|c|c|c|}
\hline \multirow{3}{*}{$\begin{array}{l}\text { Individual } \\
\text { E. } 1921\end{array}$} & \multicolumn{6}{|c|}{$\begin{array}{l}\text { Chiasma frequency within the quadrivalent } \\
\text { Region }\end{array}$} & \multicolumn{5}{|c|}{$\begin{array}{c}\text { Interference relationships } \chi^{2} \text { values) } \\
\text { Region pairs }\end{array}$} \\
\hline & 1 & II & 111 & IV & Total & N. cells & I/III & $1 / 11$ & & II/III & \\
\hline & 0.511 & $1 \cdot 307$ & $0 \cdot 667$ & 0.951 & $3.44 \pm 0.06$ & 225 & $\begin{array}{l}8 \cdot 55 * * * \\
\text { negative }\end{array}$ & $1 \cdot 52$ & N.S. & $0 \cdot 38$ & N.S. \\
\hline U3 & 0.358 & $1 \cdot 448$ & $0 \cdot 567$ & 0.985 & $3 \cdot 36 \pm 0 \cdot 07$ & 134 & $\begin{array}{l}4 \cdot 41 * \\
\text { negative }\end{array}$ & $\begin{array}{l}3 \cdot 96 \\
\text { posit }\end{array}$ & * & $1 \cdot 13$ & N.S. \\
\hline U9 & $0 \cdot 485$ & 1.400 & 0.764 & 0.988 & $3.64 \pm 0.06$ & 165 & $3 \cdot 24 \quad$ N.S. & 0.91 & N.S. & 0.81 & N.S. \\
\hline U11 & 0.443 & $1 \cdot 399$ & 0.696 & 0.918 & $3.46 \pm 0.07$ & 158 & $\begin{array}{l}15 \cdot 39 * * * \\
\text { negative }\end{array}$ & $1 \cdot 71$ & N.S. & $2 \cdot 02$ & N.S. \\
\hline
\end{tabular}

$* p<0.05$

$* * * p<0.005$

both standard homozygotes and the Al heterozygote. In this case, the mean chiasma frequency is slightly reduced: from 1.00 in normal individuals to 0.928 in the interchange (regions I+IV). This effect is remarkable since $\mathrm{M} 9$ bivalent is invariably

Table 5(a)-(c) Pairing configurations at pachytene. 5(a) A1 heterozygote. 5(b) E2411 heterozygote. 5(c) E.1921 heterozygote. $(+)$ paired, $(-)$ unpaired

\begin{tabular}{lrrrrr}
\hline & \multicolumn{3}{c}{ Region } & & \\
I & II & III & IV & N. cells & $\%$ \\
\hline+ & + & + & + & 68 & $70 \cdot 1$ \\
- & + & + & + & 20 & $20 \cdot 6$ \\
+ & + & + & - & 5 & $5 \cdot 1$ \\
- & + & + & - & 4 & $4 \cdot 2$ \\
+ & + & + & + & 52 & $88 \cdot 1$ \\
- & + & + & + & 3 & $5 \cdot 1$ \\
+ & + & - & + & 2 & $3 \cdot 4$ \\
- & + & - & + & 2 & $3 \cdot 4$ \\
+ & + & + & + & 60 & $82 \cdot 2$ \\
- & + & + & + & 9 & $12 \cdot 4$ \\
+ & + & - & + & 2 & $2 \cdot 7$ \\
+ & + & + & - & 2 & $2 \cdot 7$ \\
\hline
\end{tabular}

monochiasmatic - so that it shows complete positive interference-over the whole range of chiasma frequency variation found in standard homozygotes. Unexpectedly, this decrease is accompanied by a reduction in the interference between the M9 regions belonging to different arms of the translocation (see fig. 1(a)) since two chiasmata are formed in 12 per cent of the cells. Reductions in interference have been reported associated with the changes in pairing partner that occur in translocation heterozygotes (Parker et al., 1982). Indeed in the M6 chromosome of Euchorthippus involved in the translocation described by Arana et al. (1980) a decrease in interference similar to that observed in the M9 chromosome of Eyprepocnemis was found. However, in the M6 chromosome of Euchorthippus the mean chiasma frequency increased as well and the two regions lying on either side of the breakpoint proved to be independent of each other in the formation of chiasmata. On the contrary, the M9 chromosome of Eyprepocnemis shows a slight but significant reduction in its mean chiasma frequency and the parallel reduction in interference does not make the two regions 

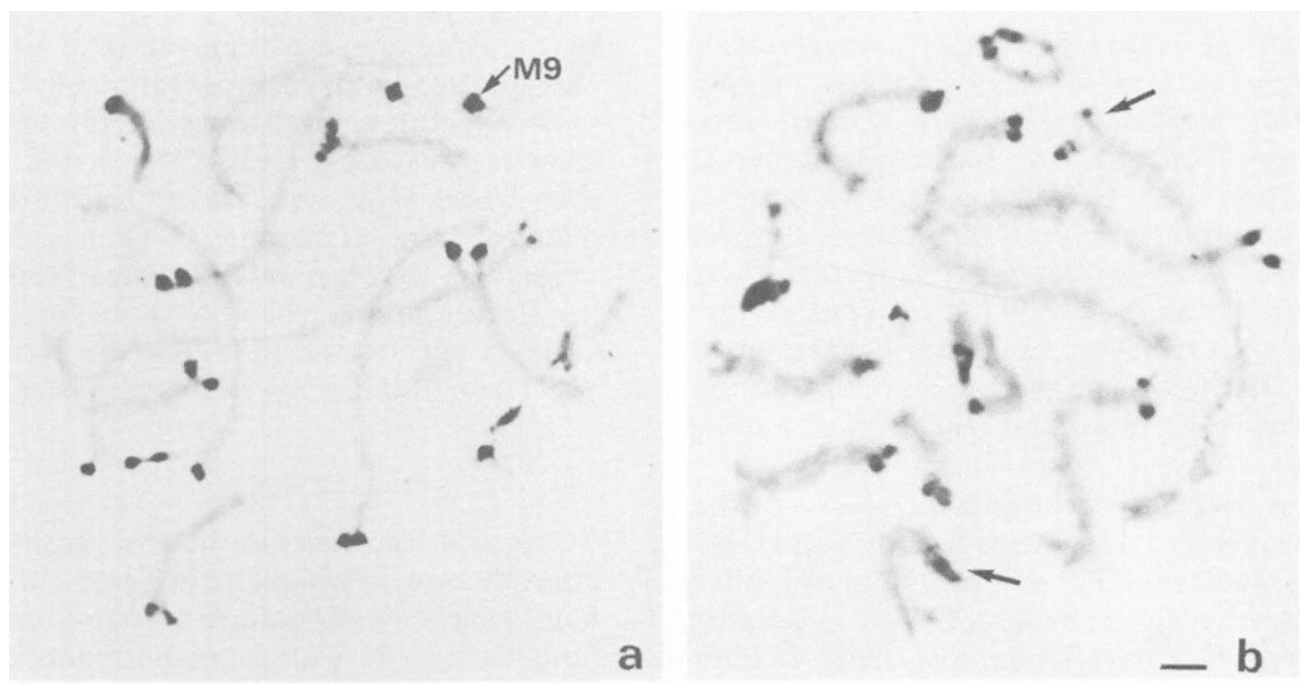

Figure 3 Synaptic failure in the interchanges at pachytene: 3(a) A1. Regions I and IV appear unpaired giving rise to a non-translocated M9 univalent (arrowed). 3(b) U11 (1921 translocation). Regions I and III fail to pair, hence no pachytene cross is formed. The resulting heteromorphic bivalents are arrowed. $\mathrm{Bar}=5 \mu \mathrm{m}$.

separated by the breakpoint totally independent (regions I and IV, table 1), since they still keep a certain level of positive interference between them.

The fact that both chiasma frequency and interference are reduced in M9 chromosome when involved in A1 interchange implies that the effect of rearrangement cannot be explained only through a modification of total chiasma frequency but also of chiasma distribution.

A reduction in mean chiasma frequency was observed in the $\mathrm{M}$ chromosome carrying a pericentric C-band involved in the E1911 interchange. Here the mean chiasma frequency is 0.85 whereas in standard homozygotes $\mathbf{M}$ group chromosomes form at least one and sometimes two chiasmata. In this translocation region $I$ is always unpaired (see fig. 1(b)) and region IV forms a maximum of one chiasma so the overall reduction of mean chiasma frequency is simply achieved by failure of chiasmata all along this chromosome. If pairing in E. plorans follows a pattern similar to that described for other grasshopper species such as Locusta (Moens, 1969; Counce and Meyer, 1973), starting at both the centromeric and non centromeric ends of the chromosomes, then the extremely proximal position of the breakpoint in E1911 individual-between the centromeric heterochromatin and the pericentric C-band (see figs. 1 (c) and 2(d)-may disturb pairing initiation in this arm, making it unable to pair and consequently to undergo crossing-over formation.

\section{Chiasma interference within the interchanges}

When analysing the interference relationships between the different translocation arms both results, independence or dependence (interference) were found and, in addition, deviations from independence could occur in two ways following the pattern proposed by Sybenga i.e., positive interference between adjacent regions and negative between opposite ones. These results are in contrast to those obtained in the L3-M6 interchange of Euchorthippus described previously (Arana et $a l ., 1980)$. In this case, the breakpoint seemed to act as a barrier for interference, the different translocation arms being independent from one another. Under the assumption of Sybenga's model, a number of factors could account for such a behaviour; first of all failure of pairing around the breakpoint might be less severe in this translocation, secondly the chromosome arms may be long enough to show a compensatory effect of chiasma formation in normally paired regions far apart from the breakpoint similar to that described by Grell (1962) in Drosophila inversion heterozygotes, and thirdly a high frequency of chiasmata in the individual bearing the translocation would mask the effect of asynapsis. The latter cause could explain why a given translocation may show different interference relationships in different individuals, as happens in the E1921 offspring. Here U9, with the highest mean chiasma frequency 
in the interchange, shows lack of interference between all the region pairs tested whereas U3, with the lowest mean chiasma frequency, shows simultaneous positive and negative interference relationships. Indeed, in further generations obtained from these $U$ males, a negative correlation has been found between the individual mean chiasma frequency within the quadrivalent and the interference showed by it (Arana et al., in preparation). Nevertheless, different behaviour or effectivity in interchange pairing cannot be discarded as a cause for the variable results found among individuals.

The lack of negative interference between the opposite translocated segments II and IV in E1911 (table 2) may have a different explanation similar to that proposed by Sybenga (1975) for a translocation in Acrida lata (Sannomiya, 1968). In both cases a short interstitial region never pairs so that it leaves the translocated segments free to move around until they have completed their pairing.

On another hand, Sybenga's model postulated the appearance of simultaneous positive and negative interference. However in the translocation heterozygotes studied here positive or negative interference can appear accompanied by independence in other interchange arms. This point is best illustrated in the E2411 translocation in which both translocated segments II and IV invariably form one chiasma each yet there is detectable negative interference between interstitial regions I and III (table 3 ). This result is not a priori illogical since actual formation of chiasmata is not a direct reflection of pairing so translocated segments may indeed influence the interstitial ones at the time of synapsis without any effect on their own chiasma formation. Comparable observations were reported by Sybenga (1970) in rye interchanges in which negative interference between interstitial segments could appear in metaphase.I bivalent pairs formed by the translocation chromosomes although in this configuration type translocated segments had no chiasmata.

In addition, as no chiasma position measurements were carried out in our material, the existence of a compensatory effect for the pairing failure around the breakpoint in the most extreme regions of the quadrivalent arms cannot be discarded. The final result of such asynapsis could be a shift in chiasma position towards the end of the arms, the total number of chiasmata in the whole region remaining constant. This effect should only be accomplished within long segments; in the short segments pairing failure may affect the whole length and indeed, unpaired short regions have been observed at pachytene (figs. 3(a), (b). If pairing is complete the segment may not be long enough to show any compensatory effect. Our data fit this hypothesis well since interference between short regions is more easily detectable, appearing even where long quadrivalent arms are independent of the remaining regions. Again, the differences between individuals carrying the same translocation might be attributable either to variation in the degree to which the compensatory effect is achieved or to actual differences in pairing.

\section{Concluding remarks}

Reciprocal translocation heterozygosity seems to affect the way in which the involved chromosomes pair, since synaptic failure is found and, in addition, it affects the shortest regions more drastically. Then, as far as pairing has a reflection in crossingover, the effect of the rearrangement on the chromosomes involved might be responsible for the changes in chiasma formation observed in them. On the other hand, the interference relationships found between the different arms of the interchange follow Sybenga's model, even though the chromosome shapes and pairing patterns in grasshoppers are different from those of rye. In the model pairing behaviour is held to account for the positive and negative interference relationships within quadrivalents; our data seem to fit this hypothesis well. Exceptions to the pattern may be explained by the existence of a compensatory effect for synaptic failure. Chiasma formation would only be accomplished in long segments and would mask the interference effect to a variable extent depending on the individual chiasma frequency. Nevertheless, actual differences in interchange pairing behaviour cannot be ruled out.

Acknowledgement This work was partially supported by a grant of the Comisión Asesora de Investigación Científica y Técnica de España.

\section{REFERENCES}

ARANA, P, SANTOS, J. L. AND CilRAldez, R. 1980. Chiasma interference and centromere co-orientation in a spontaneous translocation heterozygote of Euchorthippus pulvinatus gallicus (Acrididae; Orhtoptera). Chromosoma, 78, 327-340.

ARANA, P., HENRIQUES-GIL, N. AND SANTOS, J. L. 1987. Orientation behaviour of interchanges forming chiasmata in interstitial regions: A cytological approach. Heredity, $58,15-24$.

BLOOM, w. L. 1974. Origin of reciprocal translocations and their effect in Clarkia speciosa. Chromosoma, 49, 61-76. 
DE BOER, P. AND VAN BEEK, M. E. A. B. 1982. Meiosis in T70H translocation trisomic male mice. Chromosoma, 87, 303313.

DE BOER, P. AND VAN DER HOEVEN, F. A. 1977. Son-sire regression based heritability estimates of chiasma frequency, using $\mathrm{T} 70 \mathrm{H}$ mouse translocation heterozygotes, and the relation between univalence, chiasma frequency and sperm production. Heredity, 39, 335-343.

COUNCE, S. J. AND MEYER, F. G. 1973. Differentiation of the synaptonemal complex and the kinetochore in Locusta spermatocytes studied by whole mount electron microscopy. Chromosoma, 44, 231-253.

DIEZ, M. AND PUERTAS, M. J. 1984. Interchromosome effects on chiasma distribution due to translocation in Locusta migratoria. Genetica, 63, 189-194.

EICHER, E. M. AND GREEN, M. C. 1972. The T6 translocation in the mouse: its use in trisomy mapping, centromere localization, and cytological identification of linkage group III. Genetics, 71, 621-632.

FOMINAYA, A. AND JOUVE, N. 1986. Analysis of interference in a double heterozygote of wheat (Triticun aestivum L.). Heredity, 56, 1-6.

GOSALVEZ, J., LOPEZ-FERNANDEZ, C. AND GARCIALAFUENTE, R. 1982. A spontaneous translocation heterozygote involving centromere regions in Gomphocerus sibiricus (L.) Chromosoma, 86, 49-57.

GRELL, R. F. 1962. A new model for secondary nondisjunction: the role of distributive pairing. Genetics, 47, 1737-1754.

HAWLEY, R. S. 1980. Chromosomal sites necessary for normal levels of meiotic recombination in Drosophila melanogaster. I. Evidence for and mapping of the sites. Genetics, 94, 625-646.
HEWITT, G. M. 1967. An interchange which raises chiasma frequency. Chromosoma, 21, 285-295.

HOLM, P. B. AND RASMUSSEN, S. W. 1978. Human meiosis. III. Electron microscopial analysis of chromosome pairing in an individual with a balanced translocation $46, X Y, t\left(5 p^{-}\right.$, $22 p^{+}$). Carlsberg Res. Commun., 43, 329-350.

JOHN, B. AND HEWITT, G. M. 1963. A spontaneous interchange in Chorthippus brunneus with extensive chiasma formation in an interstitial segment. Chromosoma, 14, 638-650.

MOENS, P. B. 1969. The fine structure of meiotic chromosome polarization and pairing in Locusta migratoria spermatocytes. Chromosoma, 28, 1-25.

PARKER, J. S., PALMER, R. W., WHITEHORN, M. A. F. AND EDGAR, L. A. 1982. Chiasma frequency effects of structural chromosome change. Chromosoma, 85, 673-686.

RICKARDS, G. K. 1964. Some theoretical aspects of selective segregation in interchange complexes. Chromosoma, 15, $140-155$.

SANNOMIYA, M. 1968. Chiasma studies in structural hybrids. X. Further studies in Acrida lata. Jap. J. Genet., 43, 103-108.

SOUTHERN, D. I. 1967. Spontaneoas chromosome mutations in Truxaline grasshoppers. Chromosoma, 22, 241-257.

SYBENGA, J. 1970. Simultaneous negative and positive chiasma interference across the breakpoint in interchange heterozygotes. Genetica, 41, 209-230.

SYBENGA, J. 1975. Meiotic configurations, Springer Verlag.

SYBENGA, J. AND MASTENBROEK, I. 1980. Combined gentical and cytological analysis of positive and negative interference in an interchange heterozygote of rye (Secale cereale L.) Heredity, 44, 83-92. 